\title{
LICHENS OF THE SUPRAŚL TOWN (PODLASIE, NORTH-EASTERN POLAND)
}

\author{
AnNa Matwiejuk \\ A. Matwiejuk, Institute of Biology, University of Białystok, Świerkowa 20 B, 15-950 Białystok, Poland, \\ e-mail: matwiej@uwb.edu.pl \\ (Received: April 8, 2015. Accepted: June 15, 2015)

\begin{abstract}
A paper presents a list of 89 species found in Supraśl town (Podlasie, NE Poland). The investigations in the area of Supraśl were carried out in 2013-2014, on 41 research stands. Eighteen species are considered to be threatened in Poland. The lichens occur on all substrate types: soil, decaying wood, bark of all trees and shrubs species, boulders, concrete, foundation, mortar, plaster and bryophytes. Valuable components of the lichen biota belong mostly to the group of threatened species not only on the regional, but also on the national scale. There are: Anaptychia ciliaris, Pleurosticta acetabulum, Ramalina fastigiata and others.
\end{abstract}

KEY WORDs: lichens, distribution, urban area, north-eastern Poland

\section{INTRODUCTION}

The lichen biota of the Supraśl town has not got a comprehensive study to date. Investigations on lichens in Poland have been carried out in a large number of big and small towns, frequently of a health-resort character, situated in the lowlands as well as the mountains. On the territory of Poland lichen biota has been compiled for big cities, such as Lublin (RYDZAK 1953), Radom (CIeśLIŃsKi 1974), Warszawa (Zimny \& KuciŃsKa 1974), Kielce (Toborowicz 1976), Kraków (KiszKa 1977), Słupsk (ŚPIEWAKOWsKi \& IzYDOREK 1981), Gdańsk, Sopot, Gdynia (FatTynowicz et al. 1991), Rzeszów (PustelniaK 1991), Przemyśl (KiszKa 1999) Olsztyn (KubiaK 2005), and Białystok (MATwiejuK 2007). However, data concerning small towns populated by up to a few thousand inhabitants are not numerous, e.g. for Muszyna, Wisła (RYDZAK 1956a), Wołczyn (RYDZAK 1956b), Białowieża (RYDZaK 1957, Matwiejuk 2011), Duszniki Zdrój, Polanica Zdrój (RydzaK 1959), Limanowa (JaGiełto 1983), Drezdenko (LipNicki 1984), Ciechanowiec (MatwiejuK \& Kolanko 2007), Mielnik (Matwiejuk 2008), Boćki (Matwiejuk 2009a), Drohiczyn (Matwiejuk 2009b), Narew (Matwiejuk \& KorobKiewicz 2012), Ełk (MATWIEJUK \& WójTowicz 2013).

The aim of the study was to present flora documentation illustrating biodiversity of lichen biota in the area of the town of Supraśl, taking into consideration the habitat conditions of species.

\section{MATERIALS AND METHODS}

Fieldwork research. The investigations in the area of Supraśl were carried out in 2013-2014, on 41 research stands. These stands were established within different places. A habitat was considered a stand within which the census of lichens was made from all available substrates. The epiphytic lichens were examined for individual trees with a diameter of trunks $>30 \mathrm{~cm}$. At each stand at least five trees were monitored. At each location all species of lichens on the bark of trees from the base up to $2.5 \mathrm{~m}$ were recorded.

Taxonomic identification. For most taxa, taxonomic identification was carried out in laboratory conditions. The collected specimens were determined on the basis of morphological and anatomical (macroscopic and microscopic analysis). Lichens were also determined based on the presence of secondary metabolites - test reactions. Thin-layer chromatography (TLC) was used to identify the types of secondary metabolites, mainly from the genus Cladonia, Lepraria and Rhizocarpon. This method was conducted in accordance with the guidelines from Orange et al. (2001). The species have been named according to DieDERICH et al. (2014) and species Melanelixia (HAwKsworth et al. 2008, 2011), Lecanora saxicola to Laundon (2010), Circinaria calcarea, C. cinerea to NorDIN et al. (2010) and Calogaya decipiens, C. pusilla, Flavoplaca citrina, F. oasis, Polycauliona polycarpa, Rusavskia 
elegans to Arup et al. (2013). The lichen material has been deposited at the Herbarium of the Institute of Biology, University of Białystok.

\section{STUDY AREA}

Supraśl $\left(53^{\circ} 12^{\prime} 40^{\prime \prime} \mathrm{N} 23^{\circ} 20^{\prime} 15^{\prime \prime} \mathrm{E}\right)$ is a spa town in the Podlaskie Voivodeship, in north-eastern Poland, in Białystok County, about $15 \mathrm{~km}$ northeast of Białystok. It is the seat of Supraśl commune. Its population numbers 4,673 (2013). The total area of Supraśl is $5.68 \mathrm{~km}^{2}$ (2.19 sq mi).

Supraśl lies on a wide clearing in the heart of the Knyszyn Forest (Puszcza Knyszyńska). The Knyszyn Forest Landscape Park is Poland's largest protected area of this kind. This postglacial area, crisscrossed by streams and springs, is the home of wolves, deer, European bison, roe deer, foxes, lynx, badgers. It has a 500-year-long history, in which various cultural and religious influences interweave - Catholic, Orthodox and Uniate.

In Supraśl, the climate is cold and temperate. It is a town with a significant rainfall and even in the driest month there is a lot of rain. Precipitation averages $571 \mathrm{~mm}$. The average annual temperature in Supraśl is $6.9^{\circ} \mathrm{C}$. In January, which is the coldest month, temperatures drop to $-6.0^{\circ} \mathrm{C}$ and in July - the hottest month - they average $17.5^{\circ} \mathrm{C}$ (KoZłOWSKA-SZCZĘSNA et al. 1995, GÓRNIAK 2000).

\section{RESULTS}

On the territory of Supraśl 89 lichen species of 46 genera have been recorded. The most abundant numbers of species represented here are those of genera Lecanora (16), Physcia (6) and Cladonia, Ramalina (4).

Lichens occur on all substrata likely to be colonized - on the bark of deciduous and coniferous trees and shrubs, wooden constructions, soil, stones, concrete, mortar, plaster, metal, bryophytes and other fungi.

Lichens are represented by all morphological forms. In the biota of the investigated area, the most dominant lichens are the ones which form different types of crustose thalli. They make up 55\% of the overall number of the species. They can be found in shaded and moist places. Amongst the crustose lichens there is a significant number of pioneer species colonizing anthropogenic rock substrata, smooth tree bark and freshly-exposed soil. The second largest group consists of foliose lichens (29\%). Lichens with fruticose thalli are the less numerous group comprising $8 \%$ of the overall number of biota. The participation of lichens from other morphological groups is insignificant and amounts to around $1-4 \%$.

Epiphytes. The bark of trees (33 species) constitutes a substratum for numerous lichens. Out of 89 lichen species recorded in the area under study, 57 grew on tree bark, including 19 obligatory epiphytes. Roadside trees and trees growing in green areas dominate in Supraśl. The richest lichen biota has been recorded on the bark of deciduous trees - Tilia cordata (41 species), Acer platanoides (35), Fraxinus excelsior (23), Quercus rubra (22), Carpinus betulus (20), Salix alba and Aesculus hippocastanum (19). Among the coniferous phorophytes Larix decidua prevails, its bark showed the presence of 13 species. A small variation was also observed on the bark of Picea abies (12 species), Abies alba and Pinus sylvestris (11).

The most numerous in species is genus Lecanora (9 species). The following species were found on the largest number of phorophytes: Xanthoria parietina (29), Parmelia sulcata (26), Hypogymnia physodes (24), Phlyctis argena (21), Melanohalea exasperatula (18) and Evernia prunastri (17). The group of species attached to one phorophyte includes: Arthonia ruana, Lecanora symmicta (Sorbus aucuparia), Chaenotheca chrysocephala, Cladonia fimbriata, Parmeliopsis ambigua (Pinus sylvestris), Graphis scripta (Quercus robur), Lecanora hagenii, Pertusaria amara (Acer platanoides), Lecanora varia (Betula pendula), Phaeophyacia nigricans (Rhus typhina), Tuckermanopsis sepincola (Tilia cordata).

Lichens with crustose and foliose thalli (39\%) dominate among the epiphytic lichens. The next group of lichens is represented by fruticose lichens $(14 \%)$ and multiform (4\%), placodioid and squamulose $(2 \%)$.

On most roadside trees (e.g. Acer platanoides, Tilia cordata), high participation in lichen biota is exhibited by nitrophilous macrolichens of genera Physcia $(P$. adscendens, $P$. dubia, P. stellaris, $P$. tenella), Physconia ( $P$. enteroxantha, $P$. grisea), Ramalina ( $R$. farinacea, $R$. fraxinea) and species Anaptychia ciliaris, Parmelina tiliacea, Xanthoria parietina.

The largest amount of stands are attained by species which colonize not only tree bark but other types of substrata as well. These species also comprise the most frequently encountered group in the lichen biota of Supraśl: Phaeophyscia orbicularis, Physcia adscendens, Xanthoria parietina.

Epixylous lichens. The second largest habitat group - 46 species, comprises wood lichens, of which six are exclusive epixylous lichen.

Typical substrates occupied by epixylous lichens in Supraśl are wooden walls, roofs of buildings, poles, fences and dying branches and trunks of trees. The most numerous is genus Lecanora. Nearly half of the epixylous lichens are crustose taxa (20 species), followed by foliose (16), fruticose and multiform taxa (4), squamulose and placodioid (1).

Most of the observed epixylous lichens are common lichenized fungi. The species registered only on wood are Cladonia macilenta, C. merochlorophaea, Lecania cyrtella, Lecanora saligna, L. umbrina, Micarea denigrata, Physcia aipolia; records of other species come also from other types of substrates. 
Epilythes. The presence of rock lichens in Supraśl is marked, inter alia, on poles, fences and walls of farm buildings. Artificial anthropogenic substrate rich in calcium carbonate is mainly inhabited by calciphilous species. Quotations of epilithic lichens also come from natural substrates of stone. Stones can be found in the town and built-up areas (walls, underpinnings and gravestones). In total, 39 species were shown on rock substrate, of which 14 are exclusive epilythes. Obligatory species include, among others, Circinaria cinerea, Lecanora polytropa, Lecidea fuscoatra.

Rich lichen biota occurs also on artificial substrata with properties resembling those of rocks, such as concrete, mortar and bricks. These become colonized by calciphilous species and also by species tolerant towards the presence of calcium carbonate, such as Calogaya decipiens, C. pusilla, Lecanora albescens, L. dispersa and Xanthoria parietina. They are accompanied by numerous nitrophilous species of family Physciaceae.

Epibryophytes. Bryophytes are colonized by seven lichen species. The exclusive species is only Mycobilimbia hypnorum.

Lichens on other substrates. Metal is colonized by 16 species and the sporocarp of the polypore fungus by nine species.

Participation of vulnerable and protected lichens. Of the 89 lichen species identified in Supraśl, 18 species have been put on the Red list of the lichens in Poland (CIEŚLIŃSKI et al. 2006), including six species in the Endangered Category - EN (Anaptychia ciliaris, Physconia perisidiosa, Pleurosticta acetabulum, Ramalina fastigiata, $R$. fraxinea, Tuckermanopsis sepincola), five species in the Vulnerable Category - VU (Melanelixia subargentifera, Parmelina tiliacea, Ramalina farinacea, $R$. pollinaria, Usnea hirta), six species in the category of Near Threatened - NT (Arthonia ruana, Evernia prunastri, Graphis scripta, Hypogymnia tubulosa, Mycobilimbia hypnorum, Physcia aipolia) and one species in the category Least Concern - LC (Psilolechia lucida), as well as one species on the Red list of threatened lichens in North-Eastern Poland (CIEŚLIŃSKI 2003), in the category Data Deficient - DD (Mycobilimbia hypnorum).

The level of threat for lichens in north-eastern Poland, compared to other regions in lowland Poland is lower, which is reflected in a small number of vulnerable lichens in Supraśl placed on the local Red list (CIEśLIŃSKi 2003) compared to the Red list of the lichens in Poland (CIEśLIŃski et al. 2006). The most threatened ecological group in Supraśl are epiphytes.

Nine $(10 \%)$ of the species given are protected in Poland (RozPORZADZZENIE... 2014) including four partially protected (Hypogymnia physodes, Ramalina farinacea, R. pollinaria, Tuckermanopsis chlorophylla) and five strict protected (Anaptychia ciliaris, Parmelina tiliacea, Ramalina fastigiata, $R$. fraxinea, Tuckermanopsis sepincola).

\section{SPECIES INDEX}

Anaptychia ciliaris (L.) Körb. - bark of Acer platanoides, Tilia cordata, Ulmus laevis; sporocarp of the fungus polypores; stands: 27, 28, 30; EN (CIEŚLIŃsKI et al. 2006), strict protection.

Arthonia ruana A. Massal. - bark of Sorbus aucuparia; stand: 34, NT (CIEśLIŃsKI et al. 2006).

Bilimbia sabuletorum (Schreb.) Arnold - brick; stand: 26.

Buellia punctata (Hoffm.) A. Massal. bark of Acer platanoides, Sorbus intermedia, Tilia platyphyllos; stone; stands: 1, 17, 26, 28, 32.

Calogaya decipiens (Arnold) Arup, Frödén \& Søchting - concrete, metal, flagstone; stands: $2,5-10,12$, 14, 15-20, 22, 27, 29, 33, 35-40.

Calogaya pusilla (A. Massal.) Arup, Frödén \& Søchting - stone, concrete, terrazzo; stands: $3,4,26,27$, 28, 33, 38.

Caloplaca teicholyta (Ach.) J. Steiner - concrete; stand: 33.

Candelariella aurella (Hoffm.) Zahlbr. - wood (fences), stone, concrete, terrazzo, metal; stands: $2,3,5-7$, $9,10,13,16,18,19,22,26-28,30,32,36-41$.

Candelariella vitellina (Hoffm.) Müll. Arg. - bryophytes, wood (fences, poles, benches), stone, concrete, metal; stands: $1-3,5,8,9,10,13,14,17-19$, 26, 27, 33, 36, 38.

Candelariella xanthostigma (Ach.) Lettau - bark of Abies alba, Acer platanoides, Aesculus hippocastanum, Carpinus betulus, Cerasus vulgaris, Fraxinus excelsior, Juglans regia, Quercus robur, Q. rubra, Salix alba, Sorbus aucuparia, Tilia cordata, T. platyphyllos, Ulmus laevis; stands: 4, 5, 7, 9, 10, 12, 13-20, 22, 25-32.

Chaenotheca chrysocephala (Ach.) Th. Fr. - bark of Pinus sylvestris; stand: 33.

Circinaria calcarea (L.) A. Nordin, Savić \& Tibell concrete; stand: 16.

Circinaria cinerea (L.) A. Nordin, Savić \& Tibell stone; stand: 33.

Cladonia coniocraea (Flörke) Spreng., nom. cons. - bark of Tilia cordata, Larix decidua, Pinus sylvestris; wood (fences, benches, stumps); stands: 10, 13, 26, 27, 33, 34, 41.

Cladonia fimbriata (L.) Fr. - bark of Pinus sylvestris; bryophytes, wood (fences, stumps, branches); stands: 13, 27, 33, 34 .

Cladonia macilenta Hoffm. - wood (fences); stand: 11.

Cladonia merochlorophaea Asahina - wood (fences, benches, stumps); stands: 13, 33, 34.

Evernia prunastri (L.) Ach. - bark of Acer platanoides, A. pseudoplatanus, Aesculus hippocastanum, Betula pendula, Carpinus betulus, Crataegus oxyacantha, Fraxinus excelsior, Larix decidua, Picea abies, Prunus avium, Quercus robur, Q. rubra, Salix alba, Sorbus aucuparia, S. intermedia, Tilia cordata, Ulmus laevis; wood (fences, crosses, stumps), stone, brick, metal; stands: 1, 4, 
5, 6, 8, 10, 12-15, 21, 22, 24-34, 41, NT (CIEŚLIŃSKI et al. 2006).

Flavoplaca citrina (Hoffm.) Arup, Frödén \& Søchting - stone, concrete, brick, terrazzo; stands: $3,4,7,13$, 16, 20, 26, 27, 30, 33, 35.

Flavoplaca oasis (A. Massal.) Arup, Frödén \& Søchting - bark of Acer platanoides, Thuja occidentalis; wood (fences), stone, concrete, terrazzo, metal, flagstone; stands: $1,2,3,4,7,10,13,18,22,23,26-28,37$, $38,41$.

Graphis scripta (L.) Ach. - bark of Quercus robur; stand: 34, NT (CIEŚLIŃsKI et al. 2006).

Hypocenomyce scalaris (Ach.) M. Choisy - bark of Abies alba, Alnus glutinosa, Larix decidua, Picea abies, Pinus sylvestris, Prunus avium, Quercus robur, Tilia cordata, T. platyphyllos; bryophytes, wood (fences, poles, benches); stands: $2,6,10,11,13,14,16,17,20,26$, 27, 30, 32-34, 36, 41.

Hypogymnia physodes (L.) Nyl. - bark of Abies alba, Acer negundo, A. platanoides, Aesculus hippocastanum, Alnus glutinosa, Betula pendula, Catalpa bignonioides, Carpinus betulus, Cerasus vulgaris, Fraxinus excelsior, Larix decidua, Malus purpurea, Picea abies, Pinus sylvestris, Prunus avium, P. domestica, Quercus robur, Q. rubra, Salix alba, Sorbus aucuparia, S. intermedia, Symphoricarpos albus, Tilia cordata, T. platyphyllos; wood (fences, benches, branches), concrete, tile, stone, metal; stands: $1,2,4-6,8,9,10,13-38,41$.

Hypogymnia tubulosa (Schaer.) Hav. - bark of Cerasus vulgaris, Larix decidua, Prunus avium, Quercus rubra, Rhus typhina, Tilia cordata; wood (fences, benches); stands: $4-6,10,14,16,26,33$, NT, partially protection.

Imshaugia aleurites (Ach.) S.L.F. Meyer - bark of Picea abies, Pinus sylvestris; stands: 30, 33, partially protection.

Lecania cyrtella (Ach.) Th. Fr. - wood (fences); stand: 8.

Lecanora albescens (Hoffm.) Flörke - concrete, brick, terazzo; stands: $2-7,9,10,12,13,15,16,18,20,22$, 26-29, 33, 35-38, 40.

Lecanora allophana Nyl. - bark of Acer platanoides, Sorbus aucuparia, S. intermedia, Tilia cordata; stands: 1, $4,5,7$.

Lecanora carpinea (L.) Vain. - bark of Acer platanoides, Aesculus hippocastanum, Fraxinus excelsior, Padus avium, Quercus robur, Sorbus acuparia, S. intermedia, Tilia cordata, T. platyphyllos; wood (fences, stumps); stands: 1, 4, 7, 13, 16, 17, 20, 24, 34.

Lecanora chlarotera Nyl. - bark of Acer platanoides, Fraxinus excelsior, Tilia cordata; stands: 16, 33, 34.

Lecanora conizaeoides Cromb. - bark of Alnus glutinosa, Pinus sylvestris, Sorbus intermedia, Tilia platyphyllos; wood (fences, benches); stands: 2, 16, 17, 33, 35, 38. Lecanora crenulata Hook. - concrete; stand: 2.

Lecanora dispersa (Pers.) Sommerf. - stone, concrete, terrazzo, metal; stands: $2-8,10,12-19,20,26-30$, $33,35-40$.
Lecanora expallens Ach. - bark of Aesculus hippocastanum, Fraxinus excelsior, Tilia cordata, T. platyphyllos; wood (fences, benches); stands: 10, 13, 18, 28, 30, 33.

Lecanora hagenii (Ach.) Ach. - bark of Acer platanoides; wood (fences, poles); stands: 26, 36, 38.

Lecanora polytropa (Hoffm.) Rabenh. - stone; stands: 10, 27.

Lecanora pulicaris (Pers.) Ach. - bark of Aesculus hippocastanum, Acer platanoides, Alnus glutinosa, Fraxinus excelsior, Sorbus aucuparia, S. intermedia, Tilia cordata, T. platyphyllos; wood (fences, benches, stumps); stands: $1,4-6,10,13,16,17,21,23,27,33,34,38$.

Lecanora saligna (Schrad.) Zahlbr. - wood (poles); stand: 36.

Lecanora saxicola (Pollich) Ach. - bryophytes, wood (fences, poles, benches), stone, concrete, asbestic tile, terrazzo, metal, flagstone; stands: 1-5, 7-10, 12-15, 18, 19, 22, 24, 26, 27, 33, 36, 38.

Lecanora symmicta (Ach.) Ach. - bark of Sorbus aucuparia; wood (fences, stumps); stands: 4, 23, 34, 38.

Lecanora umbrina (Ach.) A. Massal. - wood (fences); stand: 38 .

Lecanora varia (Hoffm.) Ach. - bark of Betula pendula; wood (fences, benches); stands: 2, 8, 10, 13, 14, 18, $19,22,33,38,41$.

Lecidea fuscoatra (L.) Ach. - stone; stand: 27.

Lecidella elaeochroma (Ach.) M. Choisy - bark of Acer negundo, A. platanoides, Aesculus hippocastanum, Padus avium, Salix alba, Sorbus intermedia, Tilia cordata; wood (fences, benches, stumps); stands: 1, 16, 21, 22, 26$-28,30,33,34$.

Lecidella stigmatea (Ach.) Hertel \& Leuckert - concrete, terazzo; stands: 4, 12, 16, 22, 27, 28, 33, 37.

Lepraria elobata Tønsberg - bark of Quercus robur; stand: 34 .

Lepraria incana (L.) Ach. - bark of Abies alba, Acer platanoides, Carpinus betulus, Fraxinus excelsior, Pinus sylvestris, Quercus robur, Q. rubra, Robinia pseudoacacia, Sorbus aucuparia, Thuja occidentalis, Tilia cordata; bryophytes, wood (branches), stone, concrete, brick, metal; stands: 4, 10, 24, 26-34.

Melanelixia glabratula (Lamy) Sandler \& Arup - bark of Quercus rubra, Rhus typhina, Sorbus intermedia, Tilia cordata; stands: 4, 10, 23, 25, 26, 33, 35.

Melanelixia subargentifera (Nyl.) O. Blanco, A. Crespo, Divakar, Essl., D. Hawksw. \& Lumbsch - bark of Fraxinus excelsior, Quercus robur; stands: 16, 26, VU (CIEŚLIŃsKi et al. 2006).

Melanohalea exasperatula (Nyl.) O. Blanco, A. Crespo, Divakar, Essl., D. Hawksw. \& Lumbsch - bark of Acer platanoides, A. pseudoplatanus, Aesculus hippocastanum, Carpinus betulus, Cerasus vulgaris, Juglans regia, Fraxinus excelsior, Larix decidua, Padus avium, Picea abies, Prunus domestica, Quercus robur, Rhus typhina, Sorbus aucuparia, S. intermedia, Tilia cordata, T. platyphyllos, Ulmus laevis; wood (fences, crosses), stone, concrete, metal; stands: 1, 3-6, 9, 10, 14, 16, 17, 21, 22, 24-28, 30, 31, 33 . 
Micarea denigrata (Fr.) Hedl. - wood (fences, poles); stands: 2, 10, 36, 38.

Mycobilimbia hypnorum (Lib.) Kalb \& Hafellner bryophytes; stand: 27, NT (CIEŚLIŃSKI et al. 2006), DD (CIEśĹIŃsKI 2003).

Parmelia sulcata Taylor - bark of Abies alba, Acer negundo, A. platanoides, Aesculus hippocastanum, Alnus glutinosa, Betula pendula, Carpinus betulus, Cerasus vulgaris, Crataegus oxyacantha, Fraxinus excelsior, Juglans regia, Larix decidua, Malus purpurea, Padus avium, Prunus avium, P. domestica, Quercus robur, Q. rubra, Rhus typhi$n a$, Salix alba, Sorbus aucuparia, S. intermedia, Syringa vulgaris, Tilia cordata, T. platyphyllos, Ulmus laevis; wood (fences, poles, benches), stone, concrete, metal; stands: 1-11, 13-16, 18-33, 35, 36, 38, 41.

Parmelina tiliacea (Hoffm.) Hale - bark of Abies alba, Acer platanoides, Aesculus hippocastanum, Carpinus betulus, Fraxinus excelsior, Quercus robur, Rhus typhina, Salix alba, Tilia cordata, T. platyphyllos, Thuja occidentalis, Ulmus laevis; sporocarp of the fungus polypores, wood (fences, stumps); stands: $1,3,9,10,13,16,18,21-$ -32, VU (CIEŚLIŃsKI et al. 2006), strict protection.

Parmeliopsis ambigua (Wulfen) Nyl. - bark of Pinus sylvestris; stand: 34 .

Pertusaria albescens (Huds.) M. Choisy \& Werner - bark of Acer platanoides, Carpinus betulus, Fraxinus excelsior, Tilia cordata, Ulmus laevis; wood (benches); stands: 13, 20, 26-31.

Pertusaria amara (Ach.) Nyl. - bark of Acer platanoides; stand: 26.

Pertusaria coccodes (Ach.) Nyl. - bark of Acer platanoides, Tilia cordata, Ulmus laevis; wood (fences); stands: 26-28.

Phaeophyscia nigricans (Flörke) Moberg - bark of Rhus typhina; stone, concrete, terrazzo; stands: 3,4 , 7, 8, 10, 13-17, 22, 26-28.

Phaeophyscia orbicularis (Neck.) Moberg - bark of Abies alba, Acer negundo, A. platanoides, Aesculus hippocastanum, Fraxinus excelsior, Juglans regia, Prunus domestica, Quercus robur, Rhus typhina, Robinia pseudoacacia, Sorbus aucuparia, Tilia cordata, T. platyphyllos; bryophytes, wood (fences, poles, benches, branches), stone, concrete, asbestic tile, terrazzo, metal; stands: 1, 3, 4, 8-10, 13, 14, 16-20, 23-28, 30, 32, $33,35-40$.

Phlyctis argena (Spreng.) Flot. - bark of Abies concolor, Acer negundo, A. platanoides, A. pseudoplatanus, Aesculus hippocastanum, Carpinus betulus, Crataegus oxyacantha, Fraxinus excelsior, Juglans regia, Larix decidua, Padus avium, Picea abies, Prunus avium, Quercus robur, Q. rubra, Salix alba, Sorbus aucuparia, Thuja occidentalis, Tilia cordata, T. platyphyllos, Ulmus laevis; sporocarp of the fungus polypores, wood (fences, stumps, branches); stands: 1, 4-7, 9, 10, 12, 13, 15-18, 20-22, 24-34.

Physcia adscendens $\mathrm{H}$. Olivier, nom. cons. - bark of Acer platanoides, Aesculus hippocastanum, Larix decidua, Padus avium, Sorbus intermedia; wood (fences), concrete; stands: 1, 14, 18, 24, 25, 36.
Physcia aipolia (Humb.) Fürnr. - wood (poles); stand: 36, NT (CIEŚLIŃSKI et al. 2006).

Physcia caesia (Hoffm.) Fürnr. - wood (fences), stone, concrete, brick, terrazzo, metal; stands: 4, 5, 8, 10, 13-16, 26, 27, 36, 38.

Physcia dubia (Hoffm.) Lettau var. dubia - bark of Abies concolor, Acer platanoides, Aesculus hippocastanum, Carpinus betulus, Cerasus vulgaris, Picea abies, Prunus domestica, Quercus robur, Rhus typhina, Sorbus intermedia, Syringa vulgaris, Thuja occidentalis, Tilia cordata, T. platyphyllos; wood (fences, crosses, benches, stumps), stone, tile, terrazzo, metal; stands: $2,4,5,8,15,17$, 19, 26-28, 30-35.

Physcia stellaris (L.) Nyl. - bark of Acer platanoides, Aesculus hippocastanum, Fraxinus excelsior, Prunus domestica, Robinia pseudoacacia, Sorbus aucuparia, S. intermedia, Syringa vulgaris, Tilia cordata; wood (poles), concrete, metal; stands: $1,4,5,6,8,13,14,16,28,30$, 35,36 .

Physcia tenella (Scop.) DC. - bark of Abies alba, Acer negundo, A. platanoides, A. pseudoplatanus, Crataegus oxyacantha, Fraxinus excelsior, Padus avium, Picea abies, Quercus robur, Rhus typina, Salix alba, Sorbus aucuparia, S. intermedia, Thuja occidentalis, Tilia cordata, T. platyphyllos; wood (fences, poles, benches, stumps), stone, concrete, metal; stands: $1,3-6,8,9,10,13-19,21$, 25-33, 35, 36.

Physconia enteroxantha (Nyl.) Poelt - bark of Acer platanoides, A. pseudoplatanus, Carpinus betulus, Fraxinus excelsior, Juglans regia, Salix alba, Tilia cordata, Ulmus laevis; sporocarp of the fungus polypores, wood (stumps), concrete; stands: 1, 10, 12, 16, 26-28, 30, $31,33$.

Physconia grisea (Lam.) Poelt - bark of Acer platanoides, Aesculus hippocastanum, Carpinus betulus, Fraxinus excelsior, Quercus robur, Salix alba, Sorbus aucuparia, Tilia cordata, T. platyphyllos, Ulmus laevis; stands: 4, 9, 12, 16-20, 22, 24, 25, 27-30, 32.

Physconia perisidiosa (Erichsen) Moberg - bark of Acer platanoides, Tilia cordata; stands: 20, 26, EN.

Pleurosticta acetabulum (Neck.) Elix \& Lumbsch bark of Acer platanoides, Carpinus betulus, Fraxinus excelsior, Tilia cordata; sporocarp of the fungus polypores, wood (stumps); stand: 28, EN (CIEśLIŃSKI et al. 2006). Porpidia soredizodes (Nyl.) J.R. Laundon - stone; stand: 33.

Pseudevernia furfuracea (L.) Zopf - bark of Abies alba, Betula pendula, Carpinus betulus, Cerasus vulgaris, Larix decidua, Pinus sylvestris, Prunus avium, Quercus robur, Sorbus aucuparia, Tilia cordata; wood (fences, crosses, benches, branches); stands: 4-6, 10, 13, 16, 21, 26$-28,30,33,34$.

Psilolechia lucida (Ach.) M. Choisy - brick; stand: 33, LC (CIEśLIŃsKi et al. 2006).

Polycauliona polycarpa (Hoffm.) Frödén, Arup \& Søchting - bark of Acer platanoides, Aesculus hippocastanum, Alnus glutinosa, Betula pendula, Carpinus betulus, Larix decidua, Malus purpurea, Padus avium, Picea abies, 
Prunus avium, P. domestica, Quercus robur, Rhus typhina, Sorbus aucuparia, S. intermedia, Tilia cordata, T. platyphyllos; sporocarp of the fungus polypores, wood (fences, poles, stumps), stone, metal; stands: 1, 3-6, $8,10,13,14,16,17,19-21,25-33,36$.

Ramalina farinacea (L.) Ach. - bark of Acer platanoides, Carpinus betulus, Larix decidua, Picea abies, Quercus robur, Tilia cordata, Ulmus laevis; sporocarp of the fungus polypores, wood (fences); stands: 26-31, VU (CIEŚLIŃSKI et al. 2006), partially protection.

Ramalina fastigiata (Pers.) Ach. - bark of Acer platanoides, Carpinus betulus; stands: 26, 28, EN (CIEŚLIŃSKI et al. 2006), strict protection.

Ramalina fraxinea (L.) Ach. - bark of Acer platanoides, Carpinus betulus, Fraxinus excelsior, Salix alba, Tilia cordata, Ulmus laevis; sporocarp of the fungus polypores, wood (stumps); stands: 16, 22, 24, 27, 28, 30, 31, EN (CIEŚLIŃSKI et al. 2006), strict protection.

Ramalina pollinaria (Westr.) Ach. - bark of Acer platanoides, Carpinus betulus, Tilia cordata; stands: 26-28, 33, VU (CIEśLIŃsKI et al. 2006), partially protection.

Rhizocarpon reductum Th. Fr. - stone; stand: 27.

Rusavskia elegans (Link) S.Y. Kondr. \& Kärnefelt concrete; stands: 3, 6, 7, 12-18, 27, 28, 35, 37-39.

Scoliciosporum chlorococcum (Stenh.) Vězda - bark of Betula pendula, Tilia cordata; wood (poles, benches, stumps); stands: 9, 33, 34, 36, 38.

Scoliciosporum umbrinum (Ach.) Arnold - stone; stand: 33 .

Tuckermanopsis chlorophylla (Willd.) Hale - bark of Quercus robur, Sorbus aucuparia, Tilia cordata; stands: 9, $10,25,32$, partially protection.

Tuckermanopsis sepincola (Ehrh.) Hale - bark of Tilia cordata; stand: 7, EN (CIEŚLIŃSKI et al. 2006), strict protection.

Usnea hirta (L.) F.H. Wigg. - bark of Acer platanoides, Pinus sylvestris; stands: 25, 33, VU (CIEŚLIŃSKI et al. 2006).

Verrucaria muralis Ach. - concrete, terrazzo; stands: 3, 26, 33.

Verrucaria nigrescens Pers. - stone, concrete, brick; stands: 10, 20, 26, 27, 40.

Xanthoria parietina (L.) Th. Fr. - bark of Abies alba, Acer negundo, A. platanoides, A. pseudoplatanus, Aesculus hippocastanum, Alnus glutinosa, Betula pendula, Carpinus betulus, Catalpa bignonioides, Crataegus oxyacantha, Fraxinus excelsior, Juglans regia, Larix decidua, Malus purpurea, Padus avium, Picea abies, Prunus avium, P. domestica, Quercus robur, Rhus typhina, Robinia pseudoacacia, Salix alba, Sorbus aucuparia, S. intermedia, Symphoricarpos albus, Syringa vulgaris, Tilia cordata, T. platyphyllos, Ulmus laevis; sporocarp of the fungus polypores, wood (fences, crosses, poles, benches, stumps, branches), stone, concrete, tile, terrazzo, metal, flagstone; stands: $1,3-6,8,9,10,12-40$.

\section{INDEX OF STANDS}

1. Białostocka street, square at the bus stop. Open area, on the road; located in the residential area of detached houses - Acer platanoides, Aesculus hippocastanum, Padus avium, Sorbus intermedia; wooden bench.

2. Wczasowa street, housing estate cottages - wooden fence, concrete poles.

3. Nowa street, housing estate cottages - Rhus typhi$n a$; wooden fences, concrete underpinning, metal fences.

4. Dolna street (to the intersection of Spółdzielcza street), housing estate cottages - Aesculus hippocastanum, Sorbus aucuparia, S. intermedia, Tilia cordata; concrete wall, concrete fence, flagstone.

5. Spółdzielcza street (to the intersection of Maria Konopnicka street), housing estate cottages - Aesculus hippocastanum, Cerasus vulgaris, Sorbus aucuparia, S. intermedia; stones, concrete poles.

6. Kościelna street, housing estate cottages, theater - Prunus avium, Rhus thypina, Sorbus aucuparia, S. intermedia; concrete poles.

7. Żwirki i Wigury street (to the intersection of Kościelna street and Osiedle Robotnicze street) - Tilia cordata; concrete fence, flagstone.

8. Osiedle Robotnicze street, housing estate cottages - wooden fence, metal fence, concrete foundations, flagstone.

9. Juliusz Słowacki street, housing estate cottages Sorbus aucuparia, Tilia cordata; concrete fence, the underpinning.

10. Józef Piłsudski street (section from Juliusz Słowacki street to Aleja Niepodległości), housing estate cottages, primary school - Quercus robur, Q. rubra, Sorbus aucuparia, Tilia cordata; wooden fences, rocks, concrete fences, foundations.

11. Krasy Las street (to Butkiewicz and Ulman streets), housing estate cottages - wooden fences.

12. Piaskowa street, housing estate cottages - Acer platanoides; wooden fences.

13. Nowy Świat street (to the intersection of Cieliczańska street), housing estate cottages, near the bus stop - Sorbus aucuparia, Tilia cordata; wooden fence, stone, concrete piles, concrete underpinning, metal fence.

14. Nowy Świat street (to Cieliczańska and Topolowa streets), housing estate cottages - Larix decidua, Tilia cordata; wooden fences, concrete fences, concrete poles.

15. Nowy Świat street (to Topolowa street), housing estate cottages, the area dates back to the edge of the forest - Tilia cordata; stones, concrete foundations, concrete pillars, brick, tile, metal fences.

16. Zielona street, housing estate cottages, "Hotel Mud Spa" - Alnus glutinosa, Fraxinus excelsior, Salix alba; wooden fences, concrete poles. 


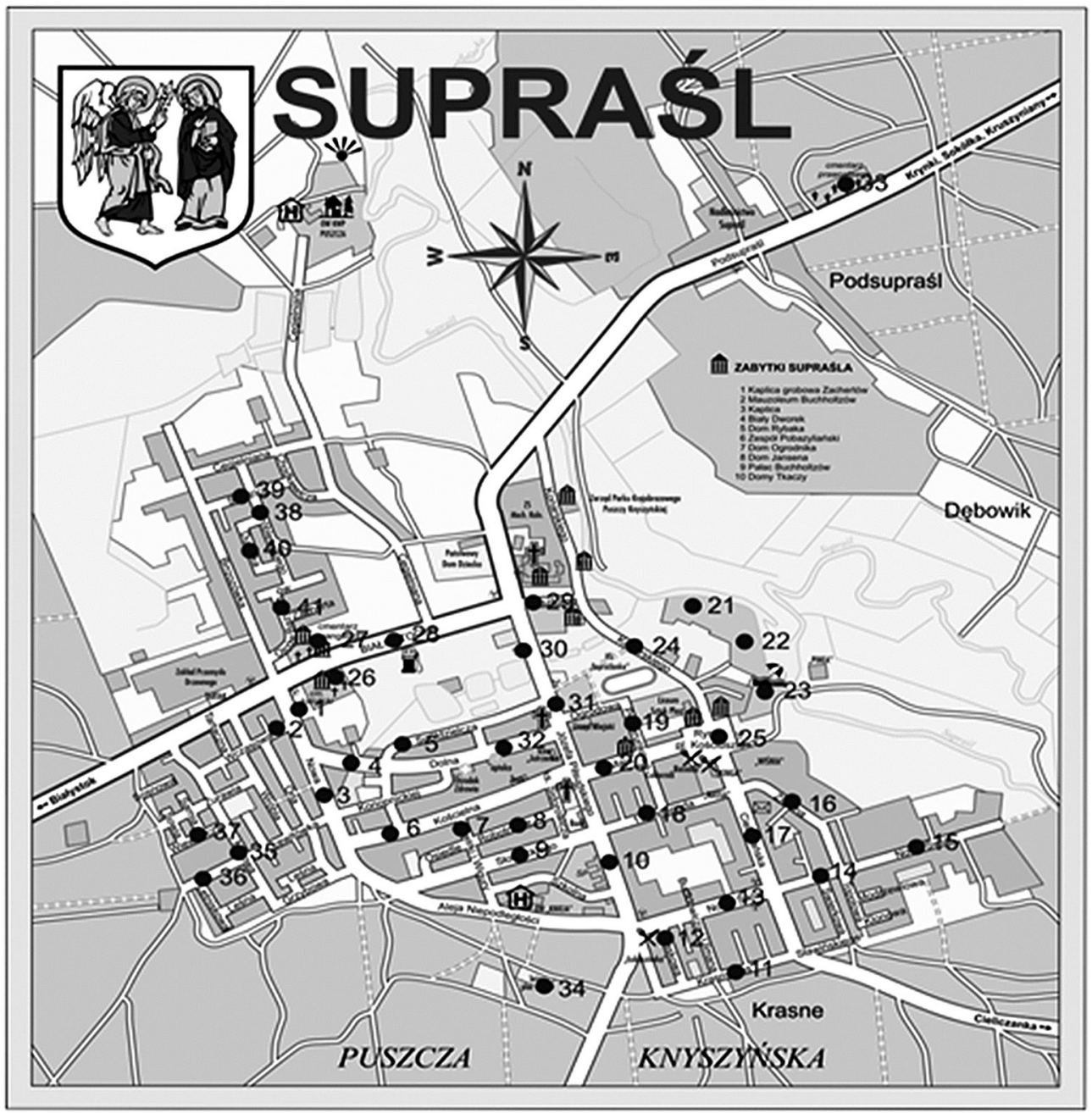

Fig. 1. Distribution of the stands in Supraśl

17. Cieliczańska street, housing estate cottages, post office, bus stop - Tilia platyphyllos, wooden fences, concrete poles.

18. 11 Listopada street, housing estate cottages Abies alba, Larix decidua, Tilia platyphyllos; wooden fence, concrete poles.

19. Posterunkowa street, housing estate cottages Tilia platyphyllos; wooden fence, concrete fences, concrete underpinning, metal fences.

20. 3 Maja street, housing estate cottages, confectionery, monument: "Weavers House" - Tilia cordata; concrete poles.

21. Beach, open area - the trees: Acer negundo, A. platanoides, Alnus glutinosa, Betula pendula, Tilia cordata.

22. Boardwalk of river Supraśl, toward to the Orthodox Monastery - Acer negundo, A. platanoides, Alnus glutinosa, Betula pendula, Tilia cordata.

23. Beach, open area - wooden fences, stones.

24. Stanisław Konarski street, housing estate cottages - Tilia cordata; wooden fences, stones; concrete poles; metal poles.

25. Tadeusz Kościuszko square - Acer platanoides, Crataegus oxyacantha, Fraxinus excelsior, Quercus robur, Tilia cordata.
26. Catholic Cemetery - Acer platanoides, Larix decidua, Pinus sylvestris, Quercus robur, Thuja occidentalis, Tilia cordata; wooden benches, crosses; stones; terrazzo tombs, brick, metal crosses.

28. Evangelical Cemetery - Acer platanoides, Aesculus hippocastanum, Fraxinus excelsior, Pinus sylvestris, Tilia cordata; wooden fences; stones; terrazzo tombstones; metal crosses.

29. Białostocka street - Acer platanoides, Carpinus betulus, Fraxinus excelsior, Prunus domestica, Tilia cordata, Ulmus laevis; sporocarp of the fungus polypores on the wood; concrete poles.

30. Klasztorna street - Tilia cordata; wood, concrete pole; metal fence.

31. Józef Piłsudski street, section from the street Białostocka for Spółdzielcza, square the road - Acer platanoides, Aesculus hippocastanum, Carpinus betulus, Picea abies, Pinus sylvestris, Robinia pseudoacacia, Tilia cordata; concrete poles.

32. Józef Piłsudski street, park - Abies concolor, Acer platanoides, A. pseudoplatanus, Carpinus betulus, Fraxinus excelsior, Picea abies, Quercus robur, Robinia pseudoacacia, Sorbus intermedia, Thuja occidentalis, Tilia cordata. 
33. Dolna street, open area, on the road; located within the housing estate houses - Acer platanoides, Carpinus betulus, Catalpa bignonioides, Juglans regia, Quercus robur, Tilia cordata.

34. Orthodox cemetery - Abies alba, Acer platanoides Pinus sylvestris, Thuja occidentalis, Tilia cordata; wooden benches; branches; stones; concrete fence; brick for the church; terrazzo tombstones; metal crosses.

35. Forest playground, forest clearing - Abies alba, Acer platanoides, Pinus sylvestris, Quercus robur, Q. rubra, Salix alba, Sorbus aucuparia; wooden stumps.

36. Majowa street (to the intersection of Żurawia street), housing estate cottages - Malus purpurea, Rhus thypina, Sorbus intermedia, Syringa vulgaris; wooden fence, concrete pole.

37. Lewitówka street (to the intersection of Majowa street), housing estate cottages - Symphoricarpos albus; wooden fence, concrete columns, flagstone.

38. Wiewiórcza street, housing estate cottages wooden fence, concrete poles.

39. Walery Wróblewski street, housing estate cottages - Betula pendula; wooden fence, concrete poles, fiber cement, metal fence, flagstone.

40. Stanisław Staszic street, housing estate cottages - concrete poles, paving stones.

41. Leon Dublak street, housing estate cottages concrete poles.

Distribution of the stands in Supraśl shown in Figure 1.

The stands are assigned to the following research areas communities: built-up areas, including downtown (stands $18,19,20,24)$, peripherals $(2,3,4,5$, $6,7,8,9,10,11,12,13,14,15,16,17,28,29,32$, $35,36,37,38,39,40,41)$ and green areas, including cemeteries $(26,27,33)$, forests $(34)$, parks $(31)$, squares $(1,25,30)$ and the beach $(21,22,23)$.

\section{DISCUSSION}

The lichen biota of Supraśl consists of 89 species of lichens. This number is relatively large considering the results of the research conducted in a sim- ilar type of towns in other parts of the country. In north-eastern Poland, in Narew 97 species of lichens were observed (MatwiejuK \& Kolanko 2007), in Mielnik - 91 (Matwiejuk 2008), in Drohiczyn - 86 (MATWIEJUK 2009b) (Table 1).

Epiphytes are the most numerous habitat group in towns. The analysed towns differ due to a significant number of species growing on the bark of trees and shrubs (Białowieża - 68 species, Limanowa -60 , Supraśl, Ciechanowiec, Boćki - 57, Drohiczyn - 44, Drezdenko - 39, Narew - 38). Their rich species variety and abundant occurrence highlights the maintenance of biocenotic structures deformed to an insignificant extent by anthropopressure.

The number of epiphytes in Polish cities is comparable to the cities such as in Ukraine. In Ukrainian cities where both suburban forests and the built-up area have been investigated and all available tree species have been monitored, only 35-60 epiphytic lichen species have been recorded, e.g. 38 species in Kherson (Khodosovtsev 1995), 48 in Lviv (KondRATYUK et al. 1991), 56 in Chernigiv (ZeLENKo 1999) and 65 in Kyiv (Dymytrova 2009).

Among the lichens colonizing secondary rock substrata the most frequently represented habitat group are calciphilous lichens bound up with concrete. These species are spread especially in city zone. The most common-place species are Lecanora albescens, $L$. dispersa, Calogaya decipiens, C. pusilla, Candelariella aurella, Phaeophyscia orbicularis, Xanthoria parietina. They frequently cover large surfaces, especially the old plaster of houses, walls and poles.

In Supraśl, as in other parts of the country, the presence of epixylous lichens is marked on the wooden buildings, fences around fields, buildings, gardens, pillars (Matwiejuk 2008, Matwiejuk 2009a, $2009 \mathrm{~b})$. The list of this group is dominated by common lichen taxa and those exhibiting a wide range of ecological spectrum, developing both on the wood and the bark of trees and on soil.

Terricolous lichens have been found outside urban settlements, primarily in forest fragments, grass-

Table. 1. Summaries of the number of species of lichens of towns of the Podlasie voivodeship of similar size to Supraśl based on literature and own data

\begin{tabular}{|c|c|c|c|c|c|c|c|c|}
\hline \multirow[b]{2}{*}{ City } & \multirow[b]{2}{*}{ Literature } & \multicolumn{7}{|c|}{ Number of species } \\
\hline & & total & epiphytes & epixylic & epilythes & epigeic & $\begin{array}{l}\text { epibrio- } \\
\text { phytes }\end{array}$ & $\begin{array}{l}\text { other sub- } \\
\text { strates }\end{array}$ \\
\hline Supraśl & & 89 & 57 & 46 & 39 & - & 7 & 9 \\
\hline Ciechanowiec & $\begin{array}{l}\text { (Matwiejuk \& } \\
\text { KolanKo 2007) }\end{array}$ & 114 & 57 & 54 & 41 & 22 & 4 & 10 \\
\hline Mielnik & (MATWIEJUK 2008) & 91 & 47 & 26 & 38 & 13 & 2 & 1 \\
\hline Boćki & (MATwIEJUK 2009a) & 118 & 57 & 41 & 38 & 31 & 2 & 15 \\
\hline Drohiczyn & (MATWIEJUK 2009b) & 86 & 44 & 6 & 32 & 15 & - & - \\
\hline Narew & $\begin{array}{l}\text { (MATWIEJUK \& Ko- } \\
\text { ROBKIEWICZ 2012) }\end{array}$ & 97 & 38 & 50 & 36 & 19 & - & 20 \\
\hline
\end{tabular}


land, on sandy wasteland situated in the vicinity of the administrative boundaries of the city and on the outskirts. In all of the towns, a group of species can be differentiated, the ones which have found optimal living conditions here.

\section{ACKNOWLEDGEMENTS}

The paper has been financially supported by the Polish Ministry of Science and Higher Education through statutory research.

\section{REFERENCES}

Arup U., SøChTing U., FröDÉN P. (2013): A new taxonomy of the family Teloschistaceae. Nordic Journal of Botany 31, 1: 16-83.

CIEŚLIŃSKI S. (1974): Flora epifityczna porostów miasta Radom. Biuletyn Kwartalny Radomskiego Towarzystwa Naukowego 11, 3/4: 169-189.

CieśLiŃSKI S. (2003): Czerwona lista porostów zagrożonych w Polsce północno-wschodniej. In: K. Czyżewska (ed.). Zagrożenie porostów w Polsce. Monographiae Botanicae 6: 91-106.

Cieśliński S., CZyżewsKa K., Fabiszewski J. (2006): Red list of the lichens in Poland. In: Z. Mirek, K. Zarzycki, W. Wojewoda, Z. Szeląg (eds). Red list of plants and fungi in Poland. W. Szafer Institute of Biology, Polish Academy of Sciences, Kraków: 71-90.

Diederich P., Ertz D., Stapper N., Sérusiaux E., van den Broeck D., van den Boom P., Ries C. (2014): The lichens and lichenicolous fungi of Belgium, Luxembourg and northern France. URL: http:// www.lichenology.info [access: 20.09.2014].

DymYtrova L. (2009): Epiphytic lichens and bryophytes as indicators of air pollution in Kyiv city (Ukraine). Folia Cryptogamica Estonica, Fasc. 46: 33-44.

FAtTynowicz W., Izydorek I., Budzbon E. (1991): The lichen flora as bioindicator of air pollution of Gdańsk, Sopot and Gdynia. Monographiae Botanicae 73: 1-52.

GóRniAK A. (2000): Klimat województwa podlaskiego. Instytut Meteorologii i Gospodarki Wodnej, Oddział w Białymstoku.

Hawksworth D.L., Blanco O., Divakar P.K., Ahti T., Crespo A. (2008): A first checklist of parmelioid and similar lichens in Europe and some adjacent territories, adopting revised generic circumscriptions and with indications of species distributions. Lichenologist 40(1): 1-21.

Hawksworth D.L., Divakar P.K., Crespo A., Ahti T. (2011): The checklist of parmelioid and similar lichens in Europe and some adjacent territories: additions and corrections. Lichenologist 43(6): 639-645.
Jagieteo M. (1983): Porosty epifityczne Limanowej. Zeszyty Naukowe Uniwersytetu Jagiellońskiego. Prace Botaniczne 11: 191-218.

Khodosovtsev A.Ye. (1995): Lichen indication assessment of Kherson city (in Russian). Konstanty 2: 52-60.

KIsZKA J. (1977): Wpływ emisji miejskich i przemysłowych na florę porostów (Lichenes) Krakowa i Puszczy Niepołomickiej. Wydawnictwo Naukowe Wyższej Szkoły Pedagogicznej w Krakowie, Prace Monograficzne 19: 5-137.

KIsZKA J. (1999): Porosty (Lichenes) oraz warunki bioekologiczne Przemyśla. Arboretum Bolestraszyce 6: 1-86.

KONDRATYUK S.Ya., KOUChERIAVYI V.O., KramaretS V.O., ZiNKo Yu.V., SiRENKO I.M. (1991): Lichen indication of air pollution in Lviv (in Ukrainian). Ukrainskyi Botanichnyi Zhurnal 48(2): 72-76.

KoZŁowsKa-SZCZĘSNa T., KrawcZYK B., BŁażejCZyK K. (1995): Warunki bioklimatyczne Supraśla. Zeszyty Instytutu Geografii i Przestrzennego Zagospodarowania Polskiej Akademii Nauk 33: 1-73.

KubiaK D. (2005): Lichens and lichenicolous fungi of Olsztyn town (NE) Poland. Acta Mycologica 40(2): 293-332.

LAUNDON J.R. (2010): Lecanora antiqua, a new saxicolous species from Great Britain, and the nomenclature and authorship of $L$. albescens, L. conferta and L. muralis. Lichenologist 42, 6: 631-635.

LIPNICKI L. (1984): Porosty miasta Drezdenka i najbliższej okolicy. Fragmenta Floristica et Geobotanica 28, 2: 221-239.

Matwiejuk A. (2007): Porosty Białegostoku. Analiza florystyczno-ekologiczna. T. 1. Wydawnictwo „Ekonomia i Środowisko”, Białystok.

Matwiejuk A. (2008): Lichens of Mielnik on river Bug (Podlasie, Eastern Poland). Nature Journal 41: 5-18.

Matwiejuk A. (2009a): Lichens of the Boćki and its surroundings in Podlasie (NE Poland). Nature Journal 42: 49-61.

Matwiejuk A. (2009b): Lichens of Drohiczyn on the Bug river (Podlasie, Eastern Poland). Roczniki Akademii Rolniczej w Poznaniu 388, Botanika-Steciana 13: 57-62.

Matwiejuk A. (2011): Anthropogenic changes of lichen biota of the Białowieża town (Podlasie, Eastern Poland). Roczniki Akademii Rolniczej w Poznaniu 390, Botanika-Steciana 15: 129-138.

Matwiejuk A., Kolanko K. (2007): Lichens of Ciechanowiec and its environs (Eastern Poland). Roczniki Akademii Rolniczej w Poznaniu 386, Botanika-Steciana 11: 85-93.

Matwiejuk A., Korobkiewicz K. (2012): Lichens of Narew and its surroudings (Podlasie, North-Eastern Poland). Roczniki Akademii Rolnicznej w Poznaniu 391, Botanika-Steciana 16: 93-100. 
Matwiejuk A., Wójtowicz E. (2013): Porosty Ełku w województwie warmińsko-mazurskim. In: I. Ciereszko, A. Bajguz (eds). Różnorodność biologiczna - od komórki do ekosystemu. Rośliny i grzyby w zmieniających się warunkach środowiska. Polskie Towarzystwo Botaniczne, Białystok 21: 291-306.

Nordin A., Savić S., Tibell L. (2010): Phylogeny and taxonomy of Aspicilia and Megasporaceae. Mycologia 102: 1339-1349.

Orange A., James P.W., White FJ. (2001): Microchemical methods for the identification of lichens. British Lichen Society, London.

Pustelniak L. (1991): Epiphytic lichens of the city Rzeszów (South-Eastern Poland). Zeszyty Naukowe Uniwersytetu Jagiellońskiego, Prace Botaniczne 22: 171-191.

RozPORZĄDZENIE Ministra Środowiska $z$ dnia 9 października 2014 r. w sprawie gatunków dziko występujących grzybów objętych ochroną. Dz.U. z 2013 r., poz. 627, z późn. zm.

RYDZAK J. (1953): Rozmieszczenie i ekologia porostów miasta Lublina. Annales Universitatis Mariae Curie-Skłodowska. Sectio C, Biologia 8, 9: 233-356.

RYDZAK J. (1956a): Wpływ małych miast na florę porostów. Part 1. Dolny Śląsk-Kluczbork-WołczynOpole-Cieszyn. Annales Universitatis Mariae Curie-Skłodowska. Sectio C, Biologia 10, 1: 1-32.

RydzaK J. (1956b): Wpływ małych miast na florę porostów. Part 2. Beskidy Zachodnie. Wisła-Ustroń-Muszyna-Iwonicz-Rymanów-Lesko. Annales
Universitatis Mariae Curie-Skłodowska. Sectio C, Biologia 10, 2: 33-66.

RYDZAK J. (1957): Wpływ małych miast na florę porostów. Part 4. Lubelszczyzna-Kieleckie-Podlaskie-Puławy-Busko-Siedlce-Białowieża. Annales Universitatis Mariae Curie-Skłodowska. Sectio C, Biologia 10, 14: 321-398.

RYDZAK J. (1959): Wpływ małych miast na florę porostów. Part 5. Kotlina Kłodzka - Kłodzko, Kudowa Zdrój, Duszniki Zdrój, Polanica Zdrój, Lądek Zdrój, Stronie Śląskie. Annales Universitatis Mariae Curie-Skłodowska. Sectio C, Biologia 11, 2: 25-50.

ŚPIEWAKOWSKI E.R., IzYDorek I. (1981): Porosty Słupska na tle warunków ekologicznych miasta. Wyższa Szkoła Pedagogiczna, Słupsk.

Tововоwicz K. (1976): Porosty miasta Kielc i najbliższej okolicy. Fragmenta Floristica Geobotanica 22, 4: 574-603.

ZELENKO S.D. (1999): Assessment of air pollution in Chernigiv using epiphytic lichens (in Ukrainian). Ukrainskyi Botanichnyi Zhurnal 56(1): 64-67.

ZimnY H., KucińsKa K. (1974): Porosty Warszawy jako biowskaźniki zaburzeń środowiska miejskiego. Przegląd Informacyjny - Zieleń Miejska 10, 1: $13-22$.

For citation: Matwiejuk A. (2015): Lichens of the Supraśl town (Podlasie, north-eastern Poland). Steciana 19(3): 133-142. doi:10.12657/steciana.019.015 